\title{
Humidity sensing of poly(diallyldimethylammonium chloride)/single-walled carbon nanotube composite films fabricated by self-assembly technique
}

\author{
Hongjun Jing, Yadong Jiang, Xiaosong Du, Huiling Tai, Guangzhong Xie \\ State Key Laboratory of Electronic Thin Films and Integrated Devices, School of Optoelectronic \\ Information, University of Electronic Science and Technology of China (UESTC), Chengdu 610054, P. \\ R. China \\ Corresponding author's e-mail address: jiangyd@uestc.edu.cn
}

\begin{abstract}
:
Single-walled carbon nanotube composite films were prepared on quartz crystal microbalance by selfassembly process, and their properties to relative humidity ranging from $20.9 \%$ to $80.2 \%$ were studied at room temperature. The treated SWNTs were characterized by Fourier transform infrared spectroscopy and X-ray photoelectron spectroscopy. Two kinds of SWNT sensors were fabricated using original SWNTs and carboxyl modified SWNTs as humidity sensitive material, respectively. The results showed that the sensitivity of the PDDA/SWNT-COOH humidity sensor was $20.23 \%$ higher than the PDDA/SWNT sensor. In contrast, the latter had a superior recovery property and the recovery time was $64 \mathrm{~s}$, while that of the PDDA/SWNT-COOH sensor was $84 \mathrm{~s}$. Both sensors had good repeatability and stability.
\end{abstract}

Key words: poly(diallyldimethylammonium chloride), single-walled carbon nanotubes, composite films, quartz crystal microbalance, self-assembly, and relative humidity

\section{Introduction}

Cheap, simple and reliable relative humidity sensors are significant in a wide variety of applications [1]. Until now, various structural and mechanistic relative humidity sensors have been studied [2]. Compared with other devices, QCM sensor has high sensitivity up to the order of nanogram magnitude [3] and good stability accordingly. On the other hand, carbon nanotubes (CNTs) hold the great promise among nanoscale materials, because of the excellent electronic, optoelectronic, mechanical properties and chemical, UV, and heat stability. Carbon nanotube thin films can be fabricated by many methods [4]. Among them, the selfassembly process does not need expensive apparatus, which reduces costs significantly. Meanwhile, it presents the fabrication of objects specified with nanometric precision [5].

Hitherto there are many studies on humidity sensors using polymer materials processed by self-assembly method. Among them, polyelectrolyte and its derivative were predominated used as the sensing materials [2]. Nevertheless, the sensors using carbon nanotubes as humidity sensitive materials by self-assembly technique are seldom reported only by Yu et al. [6] and Liu et al. [7]. Both of them used chemiresistors.

In this study, poly(diallyldimethylammonium chloride)/single-walled carbon nanotube (PDDA/SWNT) composite films were alternatively deposited on QCM to prepare the relative humidity sensor by self-assembly. Two kinds of SWNT materials were adopted, one was original SWNTs, and the other was carboxyl modified SWNTs. The effect of the precursor materials on the sensitivity, response and recovery times, reproducibility and stability, was investigated and compared.

\section{Experimental}

SWNTs, treated by concentrated $\mathrm{H}_{2} \mathrm{SO}_{4}$ and $\mathrm{HNO}_{3}(3: 1, \mathrm{v} / \mathrm{v})$, were then put into deionized water and immersed in ultrasonic baths for 30 min. The concentration of SWNTs dispersion was $0.2 \mathrm{mg} / \mathrm{ml}$. The concentration of PDDA solution was $1.0 \mathrm{wt} \%$.

The PDDA/SWNT multilayered thin films were deposited on QCM by layer-by-layer selfassembly process. Firstly, the QCM was immersed into the PDDA solution for $10 \mathrm{~min}$. Then the substrate was removed, rinsed with deionized water, and dried by nitrogen blow. The PDDA-treated substrate was then put into 
the SWNT dispersion for $20 \mathrm{~min}$, removed, rinsed with deionized water, and dried by nitrogen blow. The SWNT-terminated substrate was then dipped into the PDDA solution with $0.5 \mathrm{M} \mathrm{NaCl}$ for $10 \mathrm{~min}$. After that, the substrate was then put into SWNT dispersion for $20 \mathrm{~min}$ again. The deionized water rinsing and nitrogen drying were required for removing the excessive unstable polyelectrolytes and SWNTs on the substrate and forming stable multilayered thin films. The sequence was repeated for several times to obtain the desired number of bilayers on the substrate, designated as $(\mathrm{PDDA} / \mathrm{SWNT})_{\mathrm{n}}$ or $(\mathrm{PDDA} / \mathrm{SWNT}-\mathrm{COOH})_{\mathrm{n}}$, as shown in Fig. 1.

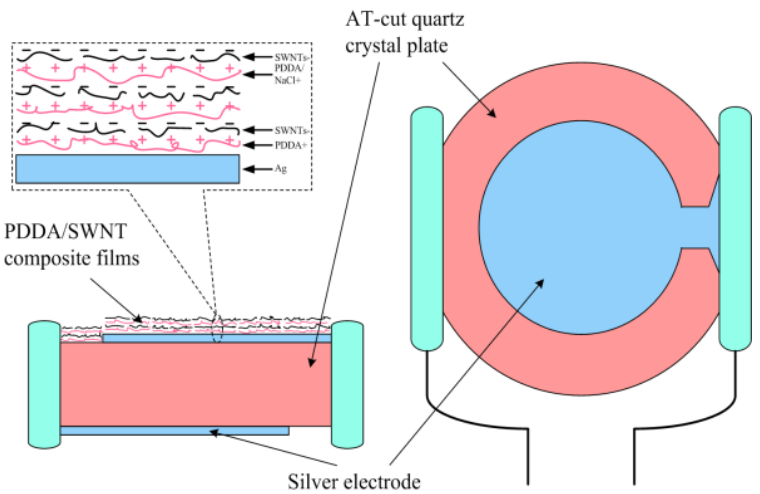

Fig. 1 Schematic diagram of fabrication process for PDDA/SWNT (PDDA/SWNT-COOH was not shown) multilayered thin films.

\section{Results and discussion}

Fig. 2 shows the FT-IR spectra $(\mathrm{KBr})$ of the modified SWNTs and SWNTs-COOH, which covers the range of $400-4000 \mathrm{~cm}^{-1}$ with a resolution of $2 \mathrm{~cm}^{-1}$. The presence of $\mathrm{O}-\mathrm{H}$ stretching band was very broad in the 3600 $2950 \mathrm{~cm}^{-1}$ region, resulting from $\mathrm{H}_{2} \mathrm{O}$ trapped in the film [8]. The adsorption bands at about 1720 $\mathrm{cm}^{-1}, 1640 \mathrm{~cm}^{-1}$, and $1400 \mathrm{~cm}^{-1}$ were attributed to the $\mathrm{C}=\mathrm{O}$ stretching vibration in the carboxylic acid group $(\mathrm{COOH})$, the carbonyl functional group $(>\mathrm{C}=\mathrm{O})$, carboxylate ion (COO-) [9], respectively. An adsorption band at $1082 \mathrm{~cm}^{-1}$ in Fig. 2(a) and $1095 \mathrm{~cm}^{-1}$ in Fig. 2(b) could be assigned to the $\mathrm{C}-\mathrm{O}$ single band. Several adsorption peaks at $3000 \mathrm{~cm}^{-1}$ range were attributed to $\mathrm{C}-\mathrm{H}$ stretching band of the $\mathrm{CH}_{\mathrm{x}}$ groups [10]. The peaks at $2376 \mathrm{~cm}^{-1}$ and 2310 $\mathrm{cm}^{-1}$ were owing to the $\mathrm{CO}_{2}$ in environmental circumstance. According to the FT-IR absorbance spectrum, $\mathrm{C}-\mathrm{O},>\mathrm{C}=\mathrm{O}, \mathrm{COOH}$, COO- and aromatic groups formed on the surfaces of carbon nanotubes after oxidation by the $\mathrm{H}_{2} \mathrm{SO}_{4}-\mathrm{HNO}_{3}$ mixture.
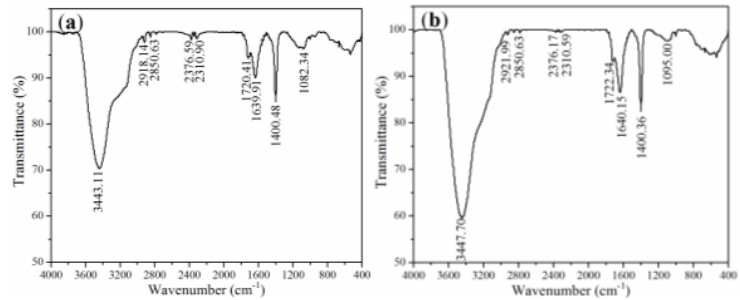

Fig. 2 FT-IR spectra of (a) modified SWNTs, (b) modified SWNTs-COOH.

The relative concentrations of the corresponding functional groups were analyzed by XPS. Fig. 3 shows the $C$ is spectrum of the modified SWNTs and SWNTs-COOH, which can be deconvoluted into the following bands: $\mathrm{sp}^{2} \mathrm{C}-\mathrm{C}$ (284.84 eV, carbon in graphite), C-O (286.1 eV, carbon singly bound to oxygen in phenols and ethers), $>\mathrm{C}=\mathrm{O}(287.5 \mathrm{eV}$, carbon doubly bound to oxygen in ketones and quinones), $\mathrm{COOH}(288.7 \mathrm{eV}$, carbon bound to two oxygens in carboxyls, carboxylic anhydrides, and esters), $\pi-\pi^{*}$ transition (290.5 $\mathrm{eV}$, i.e., the characteristic shakeup line of carbon in aromatic compounds) [11].
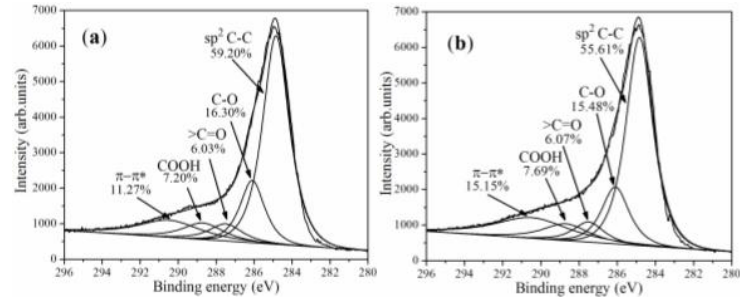

Fig. 3 XPS C 1s deconvoluted spectra of (a) modified SWNTs, (b) modified SWNTs-COOH.

$(\mathrm{PDDA} / \mathrm{SWNT})_{5}$ and (PDDA/SWNT-COOH $)_{5}$ composite films were deposited on QCM with an initial frequency shift of 737 and $679 \mathrm{~Hz}$, respectively. Fig. 4 shows the frequency changes of PDDA/SWNT and PDDA/SWNT$\mathrm{COOH} Q \mathrm{QM}$ sensors versus time when exposed to the increase and decrease of relative humidity. The two sensors behaved in a similar way when stepwise changed the relative humidity. Thus, an offset was made between the two curves to avoid overlapping. However, the PDDA/SWNT sensor responded quickly and exhibited much more flat-topped profiles at all the humidity tests. Nevertheless, it took a longer time for the PDDA/SWNT-COOH sensor to reach the steady state, especially at high humidity. The sensitivity can be defined as:

$$
\text { Sensitivity }=-\frac{f_{R H}-f_{20.9}}{f_{20.9}} \times 100 \%
$$

According to eq. (1), the sensitivities of the two sensors at different relative humidity were calculated and listed in Tab. 1. It can be seen clearly that the sensitivity of the PDDA/SWNT$\mathrm{COOH}$ sensor was much higher than the 
PDDA/SWNT sensor during all the adsorption and desorption processes. For example, the sensitivity enhancement of the PDDA/SWNT$\mathrm{COOH}$ sensor $(16.64 \%)$ was $20.23 \%$ when comparing with the PDDA/SWNT sensor $(13.84 \%)$ at the relative humidity of $80.2 \%$ in Tab. 1. When the relative humidity went back again to $20.9 \%$, the sensors did not return to their initial frequencies completely. This could be attributed to the water molecules penetrating deep into the films and the condensation of water inside them at higher relative humidity.

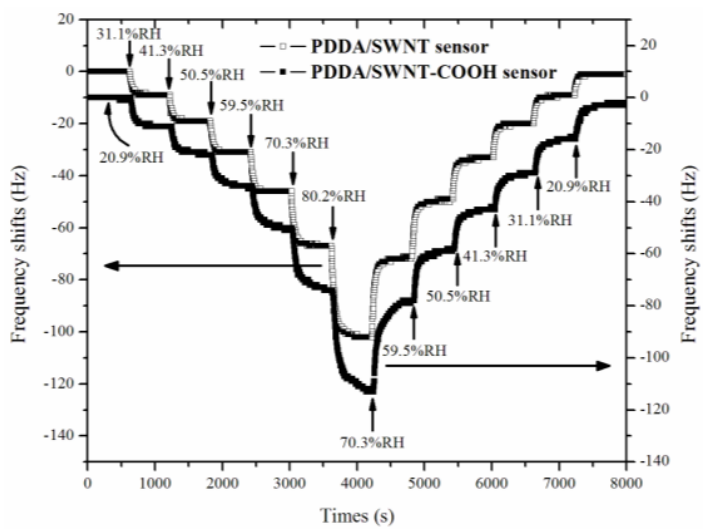

Fig. 4 Frequency changes of sensors while the relative humidity ranging from 20.9 to $80.2 \%$.

Tab. 1: Sensitivities of PDDA/SWNT and PDDA/SWNT-COOH sensors at various humidity.

\begin{tabular}{lllll}
\hline $\begin{array}{l}\text { Relative } \\
\text { humidity } \\
(\% \mathrm{RH})\end{array}$ & \multicolumn{2}{l}{ Modified SWNTs } & $\begin{array}{l}\text { Modified } \\
\text { COOH }\end{array}$ & SWNTs- \\
\cline { 2 - 5 } & Increase & Decrease & Increase & Decrease \\
\hline 31.1 & 1.22 & 1.22 & 1.62 & 2.21 \\
41.3 & 2.58 & 2.71 & 3.24 & 4.27 \\
50.5 & 4.21 & 4.48 & 5.01 & 6.33 \\
59.5 & 6.24 & 6.65 & 7.36 & 8.54 \\
70.3 & 9.09 & 9.63 & 10.90 & 11.49 \\
80.2 & 13.84 & 13.84 & 16.64 & 16.64 \\
\hline
\end{tabular}

Fig. 5 illustrates the response and recovery curves of the two QCM sensors exposed to 20.9\% RH and $80.2 \% \mathrm{RH}$ alternately. Two square wave profiles were exhibited during humidity recirculation, indicating good repeatability and stability of the sensors. It also can be seen that for the PDDA/SWNT-COOH sensor in the second and third loop, the profile was much more flat-topped as a comparison with the first loop and the corresponding curve in Fig. 4, implying that this sensor should be aged under high humidity for several times before application. The response time $t_{90}$ could decrease from $148 \mathrm{~s}$ for the first loop to $94-99 \mathrm{~s}$ for the following loop, which was very close to $t_{90}$ of the PDDA/SWNT sensor around $100 \mathrm{~s}$. However, the recovery times were recorded as
61-67 s for the PDDA/SWNT sensor and $84 \mathrm{~s}$ for the PDDA/SWNT-COOH sensor, which were not affected by the loop process.

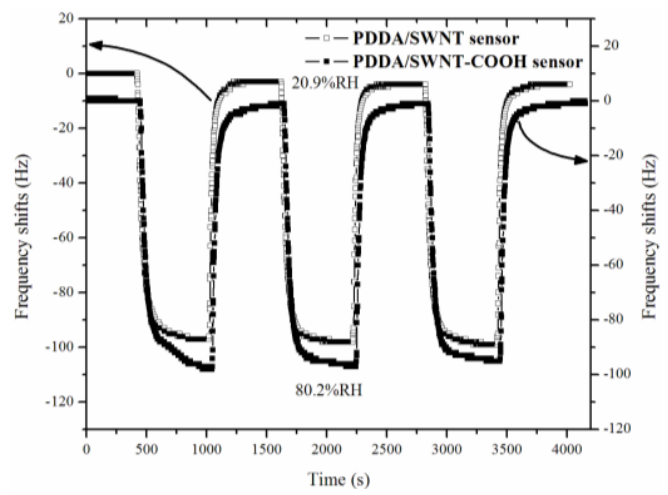

Fig. 5 Typical response and recovery curves of the two humidity sensors.

Fig. 6 shows the response and recovery times of the two sensors with the layer number ranging from 5 to 9 . The response and recovery times both increased with the increasing of the layer number. However, the response time of the PDDA/SWNT sensor was longer than the PDDA/SWNT-COOH sensor, while the recovery time was shorter than the latter, which were much more obvious when the layer numbers were more. This phenomenon implied that the SWNTs had a greater influence when the thickness of the two multilayered composite films increased.

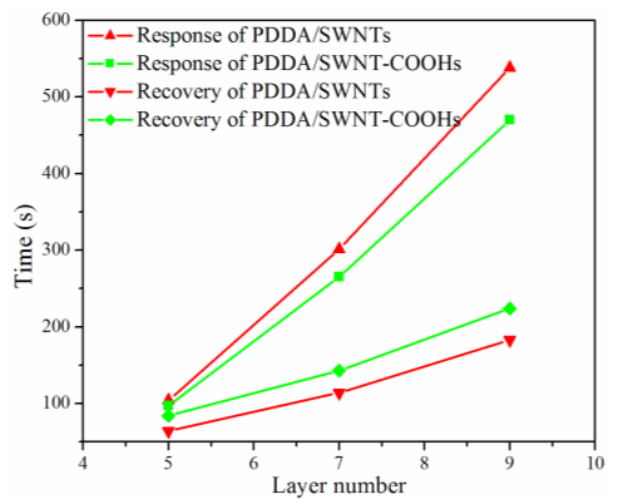

Fig. 6 Response and recovery times of humidity sensors of various layer numbers.

For a humidity QCM sensor, the frequency decrease is owing to the mass increase of adsorbed water molecules on the surface of the QCM resonator, and often expressed by the Sauerbrey equation.

According to XPS, the relative concentrations of $\mathrm{C}-\mathrm{O},>\mathrm{C}=\mathrm{O}$ and $\mathrm{COOH}$ were basically identical in modified SWNTs and SWNTS-COOH. However, the relative content of $\pi-\pi^{*}$ transitions in SWNTs-COOH was $15.15 \%$, which was much higher than that of SWNTs with $11.27 \%$. Because of more functional groups in SWNTs-COOH, the QCM humidity 
sensor could adsorb more water molecules and water clusters by forming hydrogen-bonds. Furthermore, high contents of these structures would offer greater opportunities for the cooperative effect and be more likely to form "bridges" and eventually induce condensation between the neighboring functional groups. Thus, the PDDA/SWNT-COOH QCM humidity sensor had a much higher sensitivity.

For desorption, the formed hydrogen-bonds should be broken. When more bonds were formed for the PDDA/SWNT-COOH composite films, it would consume much more time for the sensor to recover to its initial state. As a result, the recovery time of the PDDA/SWNT QCM sensor was much shorter than that of the PDDA/SWNT-COOH sensor. This phenomenon became more and more obvious when the layer number and thickness of the two multilayered thin films increased.

\section{Conclusion}

PDDA/SWNT and modified PDDA/SWNT$\mathrm{COOH}$ multilayered thin films were deposited on QCM by self-assembly technique and used for the detection of relative humidity ranging from $20.9 \%$ to $80.2 \%$. The humidity sensing properties of the two composite films were tested and compared at room temperature. The PDDA/SWNT and PDDA/SWNT-COOH sensors both had a nonlinear response to the relative humidity in the range of $20.9-80.2 \% \mathrm{RH}$. The sensitivity of the PDDA/SWNT-COOH sensor was $20.23 \%$ higher than the PDDA/SWNT sensor. The response time of the two sensors was about $100 \mathrm{~s}$ when the layer number was 5 , while the recovery times were $84 \mathrm{~s}$ and $64 \mathrm{~s}$, respectively. The response and recovery times both increased with the increasing of the layer number, but the recovery time of the PDDA/SWNT sensor was obviously shorter than the PDDA/SWNT-COOH sensor at all the layer number and thickness. Both sensors had good repeatability and stability. XPS analysis showed that the PDDA/SWNT$\mathrm{COOH}$ composite film had higher contents of $\pi$ $\pi^{*}$ transition structures. Thus, it could adsorb much more water molecules, resulting in higher sensitivity. Meanwhile, it needed much more energy to break the formed hydrogen-bonds, so the PDDA/SWNT-COOH sensor also had a longer recovery time to moisture.

\section{Acknowledgements}

This work was supported by the National Natural Science Foundation of China via Grant Nos. 60736005 and 60876050, the Foundation for Innovation Groups of NSFC via No. 61021061, and the Program for New Century
Excellent Talents in University via Grant No. NCET-08-0086.

\section{References}

[1] N. Yamazoe, Y. Shimizu, Humiditysensors: principles and applications, Sensors and Actuators 10, 379-398 (1986); doi:10.1016/02506874(86)80055-5

[2] S. Korposh, R. Selyanchyn, S.-W. Lee, Nanoassembled thin film gas sensors. IV. Masssensitive monitoring of humidityusing quartz crystal microbalance (QCM) electrodes, Sensors and Actuators B: Chemical 147,599-606 (2010); doi:10.1016/j.snb.2010.04.006

[3] I.A. Koshets, Z.I. Kazants eva, Y.M. Shirshov, S.A. Cherenok, V.I. Kalchenko, Calixarene films as sensitive coatings for QCM-based gas sensors, Sensors and Actuators B: Chemical 106,177181 (2005); doi:10.1016/j.snb.2004.05.054

[4] L. Hu, D.S. Hecht, G. Gru"ner, Carbon Nanotube Thin Films: Fabrication, Properties, and Applications, Chemical Reviews 110, 5790-5844 (2010); doi: 10.1021/cr9002962

[5] E. Winfree, F. Liu, L.A. Wenzler, N.C. Seeman, Design and self-assembly of two-dimensional DNA crystals, Nature 394, 539-544 (1998); doi:10.1038/28998

[6] H. Yu, T. Cao, L. Zhou, E. Gu, D. Yu, D. Jiang, Layer-by-Layer assemblyand humiditys ensitive behavior of poly(ethyleneimine)/multiwall carbon nanotube com posite films, Sensors and Actuators B: Chemical 119, 512-515(2006); doi:10.1016/j.snb.2005.12.048

[7] L. Liu, X. Ye, K. Wu, Z. Zhou, D. Lee, T. Cui, Humidity Sensitivity of Carbon Nanotube and Poly (Dimethyldiallylammonium Chloride) Composite Films, IEEE Sensors Journal9, 13081314 (2009); doi: 10.1109/jsen.2009.2030381

[8] M.P. Bernstein, S.A. Sandford, L.J. Allamandola, J.S. Gillette, S.J. Clemett, R.N. Zare, UV Irradiation of Polycyclic Aromatic Hydrocarbons in Ices: Production of Alcohols, Quinones, and Ethers, Science 283, 1135-1138 (1999); doi: 10.1126/s cience.283.5405.1135

[9] H.G. Agrell, J. Lindgren, A. Hagfeldt, Degradation mechanisms in a dye-sensitized solar cell studied by UV-VIS and IR spectroscopy, Solar Energy 75, 169-180 (2003); doi:10.1016/S0038092X(03)00248-2

[10] N. Kouklin, M. Tzolov, D. Straus, A. Yin, J.M. Xu, Infrared absorption properties of carbon nanotubes synthesized by chemical vapor deposition, Applied Physics Letters 85, 44634465 (2004); doi:10.1063/1.1812837

[11] S. Kundu, Y. Wang, W. Xia, M. Muhler, Thermal Stability and Reducibility of Oxygen-Containing Functional Groups on Multiwalled Carbon Nanotube Surfaces: A Quantitative HighResolution XPS and TPD/TPR Study, The Journal of Physical Chemistry C112, 1686916878 (2008); doi: 10.1021/jp804413a 